This document is the accepted manuscript version of the following article:

Chen, B., Janssens, K. G. F., \& Dunne, F. P. E. (2020). Role of geometrically necessary dislocation density in multiaxial and non-proportional fatigue crack nucleation. International Journal of Fatigue, 135, 105517 (13 pp.).

https://doi .org/10.1016/j.ijfatigue.2020.105517

\title{
Role of geometrically necessary dislocation density in multiaxial and non-proportional fatigue crack nucleation
}

\author{
Bo Chen ${ }^{1}$, Koen Janssens ${ }^{2}$, and Fionn P.E. Dunne ${ }^{1, *}$ \\ ${ }^{1}$ Department of Materials, Imperial College London, SW7 2AZ, UK \\ ${ }^{2}$ Laboratory for Nuclear Materials, Paul Scherrer Institute, CH-5232 Villigen PSI, \\ Switzerland
}

*Corresponding author: fionn.dunne@imperial.ac.uk

\begin{abstract}
Experimental and crystal plasticity modelling studies have been carried out to investigate nonproportionality and stress state effects in fatigue in a 316 stainless steel and nickel-based superalloy RR1000 which have substantial effects on fatigue life. Stored energy density has provided a reasonably consistent and unifying explanation for the experimental observations of fatigue life in axial, torsional, in-phase proportional tension and torsion, and nonproportional loading regimes. A single fatigue quantity (the critical stored energy density, equating to new surface energy) has been shown to provide good qualitative and reasonable quantitative prediction of the experimental observations of the complex loading, providing a mechanistic explanation for the fatigue behaviour. For the case where significant densities of GNDs develop (for the fine-grained nickel), the latter is found to differentiate the proportional and non-proportional fatigue lives and its contribution to the local stored energy is crucial for capturing the correct fatigue lives under the differing loadings.
\end{abstract}

\section{Introduction}

Many engineering structures are subjected to repeated thermal and mechanical loadings in service, hence fatigue is a common problem and its evaluation remains one of the major considerations in the design and reliability assessment of materials [1,2]. For many cases, fatigue failure results from complex multiaxial loadings such as non-proportional and out-ofphase loads; that is, the corresponding principal stresses change direction and their ratios change during a cycle in such loadings [3-6]. In particular, the changing of principal stress directions under non-proportional loadings is considered to contribute to fatigue life reduction of structures due to additional hardening arising from such loadings [7, 8]. Therefore, an understanding of multiaxial fatigue behaviour and non-proportional loading of structures, which is based on mechanistic behaviour, is necessary for life predictions.

Uniaxial fatigue behaviour of metals has been reasonably well quantified (if not fully understood) and described because of the relatively simple state of loading. However, limited knowledge exists for multiaxial fatigue cases, because the responses of materials and structures are affected by many factors under such loadings, including the directions of the principal 
stresses $[9,10]$, the non-proportionality of loading paths $[1,11,12]$, the ratio of stress components and mean stress [13-15], and the out-of-phase angles [6, 16]. Various multiaxial fatigue criteria for metallic alloys have been proposed to address fatigue failure and correlate fatigue lives. In general, these criteria can be classified as stress or strain based or a combination of both, critical plane and energy-based criteria. Criteria based upon von Mises and Tresca methodologies, which focus on finding equivalent fatigue damage parameters, are assumed to generate the same fatigue damage as uniaxial loading [6]. These criteria have been studied and reported to give non-conservative life under non-proportional loading regimes [7, 17]. In order to avoid this drawback, fatigue failure approaches based on critical plane and energy have been developed. For example, six differing critical plane approaches have been used to investigate the observed fatigue failures in nickel-based superalloy DD6 [18]. A number of life prediction models which incorporate strain and stress components on the critical plane have also been studied by other researchers [19-21]. In their studies, multiaxial fatigue criteria which explicitly include shear strain combined with normal strain or normal stress have been proposed. These methodologies have been evaluated for different materials and reasonably good correlation of fatigue lives has been achieved and reported [8, 22]. Earlier work has shown that the cyclic accumulated slip or plastic strain energy is closely associated with fatigue damage processes. For instance, in the study of Brown and Miller [23], they demonstrated that the shear strain energy accounts for the effect of shear plastic deformation and correlates well with multiaxial fatigue data under tension-torsion fatigue loading conditions. In the recent work of Lee et al [24] and Noban et al [25], an elastic energy term has been added into the energy parameter proposed earlier, to overcome the shortcoming that limited plasticity occurs in the high and very high cycle fatigue regimes. Much work, including that recently in [26-28], addresses strain-controlled loading histories; similarly, stress-controlled fatigue has also been assessed together with mean stress effects. While attempts to correlate accurately fatigue lives observed in test samples and components is a laudable aim, it is also appropriate to seek mechanistic understanding in fatigue with the objective that reduced damage parameters, a broader range of applicability and the ability to generalise to complex loading paths may be achieved in component design.

Previous dislocation-based work has addressed elastic stored energy criteria integrated within crystal plasticity modelling with explicit representation of microstructure in order to attempt to understand the contributions of local microstructural strain, stress and dislocation density to fatigue damage behaviour [29-31]. These methods have been able to capture the microstructural sites of crack nucleation, and to give reasonable quantitative prediction of fatigue lifetime under uniaxial loading. The work presented in this paper addresses multiaxial and non-proportional effects on the fatigue life of stainless steel 316L and nickel-based superalloy RR1000 using a dislocation-based elastic stored energy crystal plasticity approach. It is shown that a single fatigue crack nucleation quantity, the critical stored energy, in combination with microstructural crystal plasticity modelling, captures sufficient aspects of the slip and damage mechanisms to allow non-proportional and multiaxial effects on fatigue crack nucleation to be captured naturally. In addition, the role of geometrically necessary dislocations (GNDs) in non-proportional fatigue is investigated with respect to multi-slip activation and stored energy accumulation as a driver of the observed fatigue life reduction.

In the following sections, the computational framework is firstly introduced and followed by the modelling and experimental studies of proportional and non-proportional fatigue in $316 \mathrm{~L}$ steel and RR1000 nickel alloy. 


\section{Computational methodology and stored energy criterion}

In face centred cubic (FCC) crystals, there are 12 slip systems ([ $\left[\begin{array}{lll}1 & 1 & 1\end{array}\right]<\begin{array}{llllll}1 & 0\end{array}$ family) that can be activated potentially during loading. The crystal plasticity model utilised is rate-dependent and implemented in a user material subroutine (UMAT) using ABAQUS standard/explicit analysis [29-31]. The plastic velocity gradient $\mathbf{L}^{\mathrm{P}}$ is given in terms of the plastic deformation gradient $\mathbf{F}^{\mathrm{p}}$ by

$$
\mathbf{L}^{\mathrm{P}}=\dot{\mathbf{F}}^{\mathrm{p}} \mathbf{F}^{\mathrm{p}^{-1}}=\sum_{\alpha=1}^{12} \dot{\gamma}^{\alpha} \mathbf{s}^{\alpha} \otimes \mathbf{n}^{\alpha}
$$

in which $\mathbf{s}^{\alpha}$ and $\mathbf{n}^{\alpha}$ are the line vectors along slip directions and slip plane normals of slip system $\alpha$ respectively, and the slip rate on a given slip system is expressed by the slip rule

$$
\dot{\gamma}^{\alpha}=\rho_{\mathrm{ssdm}} \mathrm{b}^{2} v \exp \left(-\frac{\Delta \mathrm{F}}{\mathrm{kT}}\right) \sinh \left(\frac{\Delta \mathrm{V}}{\mathrm{kT}}\left|\tau^{\alpha}-\tau_{\mathrm{c}}{ }^{\alpha}\right|\right)
$$

where $\rho_{\text {ssdm }}$ is the mobile dislocation density, $b$ the magnitude of Burgers vector, $v$ the frequency of dislocation attempts to jump energy barriers, successful or otherwise, $\Delta \mathrm{F}$ the activation energy for dislocation escape from pinning, with corresponding activation volume $\Delta \mathrm{V}, \mathrm{k}$ the Boltzman constant, $\mathrm{T}$ the operating temperature, $\tau^{\alpha}$ the resolved shear stress for slip system $\alpha$ and $\tau_{\mathrm{c}}{ }^{\alpha}$ is the corresponding critical resolved shear stress.

In this study, a modified Taylor hardening rule is used in equation (3), where $\tau_{\mathrm{c} 0}$ is the primary critical resolved shear stress and $G_{12}$ is the in-plane shear modulus. Isotropic hardening is used in this study, such that the hardening rates of the 12 slip systems are the same.

$$
\tau_{\mathrm{c}}{ }^{\alpha}=\tau_{\mathrm{c} 0}+\mathrm{G}_{12} \mathrm{~b} \sqrt{\rho_{\mathrm{SSD}}+\rho_{\mathrm{GND}}}
$$

The development of sessile SSDs provides hardening through shear resistance on each slip system. Here, the evolution of $\rho_{\mathrm{SSD}}$ is computed from the accumulated plastic strain thus giving rise to isotropic hardening

$$
\dot{\rho}_{\mathrm{SSD}}=\lambda \dot{\mathrm{p}}
$$

where $\lambda$ provides the rate of accumulation and is determined from experiments to ensure appropriate polycrystal hardening, where $\dot{p}$ is the effective plastic strain rate.

The existence of GNDs is the result of strain gradients to accommodate the lattice curvatures. The Nye dislocation tensor $\boldsymbol{\Lambda}$ is utilized to compute the components of GNDs from

$$
\boldsymbol{\Lambda}=\operatorname{curl}\left(\mathbf{F}^{\mathbf{P}}\right)=\sum_{\alpha=1}^{12} \rho_{\mathrm{Gs}}^{\alpha} \mathbf{b}^{\alpha} \otimes \mathbf{m}^{\alpha}+\rho_{\mathrm{Get}}^{\alpha} \mathbf{b}^{\alpha} \otimes \mathbf{t}^{\alpha}+\rho_{\mathrm{Gen}}^{\alpha} \mathbf{b}^{\alpha} \otimes \mathbf{n}^{\boldsymbol{\alpha}}
$$

where $\rho_{\text {Gs }}^{\alpha}$ is the screw component while $\rho_{\text {Get }}^{\alpha}$ and $\rho_{\text {Gen }}^{\alpha}$ are edge components on slip system $\alpha$. $\mathbf{b}^{\alpha}$ is the burgers vector on slip system $\alpha$, and $\mathbf{m}^{\alpha}, \mathbf{t}^{\boldsymbol{\alpha}}$ and $\mathbf{n}^{\boldsymbol{\alpha}}$ form an orthogonal unit vector set. The 12 screw and 24 edge dislocation components need to be calculated from Nye's dislocation tensor, hence equation (5) is expressed in matrix form

$$
\mathbf{A} \rho_{\mathrm{G}}=\bar{\Lambda}
$$

where $\overline{\boldsymbol{\Lambda}}$ is the column vector formed of Nye's dislocation tensor with size of $9 \times 1$, and $\mathbf{A}$ $(9 \times 36)$ contains the basis tensors $\mathbf{b}^{\alpha} \otimes \mathbf{m}^{\alpha}, \mathbf{b}^{\alpha} \otimes \mathbf{t}^{\alpha}$ and $\mathbf{b}^{\alpha} \otimes \mathbf{n}^{\alpha}$; hence the 9 rows in $\mathbf{A}$ incorporate the 9 components for each calculated tensor product, for each of the 36 GND components. Hence the 36 individual GND components cannot be determined uniquely using equation (6), and the $\mathrm{L}_{2}$-norm minimization method is utilized here to minimise the sum of the weighted squares of the resulting GND densities, which is written as

$$
\rho_{G N D}=\sqrt{\sum_{\alpha=1}^{12}\left[\left(\rho_{\mathrm{GS}}^{\alpha}\right)^{2}+\left(\rho_{\mathrm{Get}}^{\alpha}\right)^{2}+\left(\rho_{\mathrm{Gen}}^{\alpha}\right)^{2}\right]} .
$$


A stored elastic energy criterion has been proposed recently to predict fatigue behaviour of materials. The criterion is closely associated with the localization of slip, stress states and the densities of SSDs and GNDs. The determination of full-field distributions and evolutions of dislocation density, slip accumulation and stress, which are related to the stored energy, are available from the crystal plasticity model. The local elastic stored energy density is considered to be driven by dislocation pile-up and structure formation over a length scale determined by the local dislocation density. The stored energy density rate (per cycle) is determined by

$$
\dot{\mathrm{G}}=\frac{\zeta \dot{\mathrm{U}} \Delta \mathrm{V}_{\mathrm{s}}}{\Delta \mathrm{A}_{\mathrm{s}}}=\int \frac{\zeta \boldsymbol{\sigma}: \mathrm{d} \boldsymbol{\varepsilon}^{\mathrm{p}}}{\sqrt{\rho_{\mathrm{SSD}}+\rho_{\mathrm{GND}}}}
$$

in which $\dot{U}$ is the dissipated plastic energy per cycle per unit volume and $\zeta$ of this dissipated energy is stored as dislocation structures. The precise magnitude of $\zeta$ remains to be determined but is often taken to be approximately $5 \%$ of total energy associated with plastic deformation, as the empirically accepted fraction of dissipated energy emerging as heat is documented to be about $95 \%$. The storage volume $\Delta \mathrm{V}_{\mathrm{s}}$ may be expressed by

$$
\Delta \mathrm{V}_{\mathrm{s}}=\lambda^{\prime} \Delta \mathrm{A}_{\mathrm{s}}=\frac{\Delta \mathrm{A}_{\mathrm{s}}}{\sqrt{\rho_{\mathrm{SSD}}+\rho_{\mathrm{GND}}}}
$$

where $\lambda^{\prime}$ is the mean free distance of resultant dislocations and $\Delta \mathrm{A}_{\mathrm{S}}$ is the corresponding surface area. The stored energy required to drive fatigue crack nucleation is called the critical stored energy, $G_{c}$, to generate new crack surfaces and may be written in general and without assumption of a linear accumulation of cycles, $\mathrm{N}$, as

$$
\mathrm{G}_{\mathrm{c}}=\int_{\text {cycles }} \dot{\mathrm{G}} d \mathrm{~N}
$$

\section{Fatigue analysis in stainless steel 316L}

An austenitic stainless steel of grade AISI 316L is considered. The development of a representative microstructural model using CPFE modelling is based on the EBSD characterisation of the 316L specimens. Experimental fatigue tests were performed for a range of loading conditions for austenitic 316L steel and the corresponding test results provide information on fatigue life difference and scatter under various loading paths.

\subsection{Materials characterisation and mechanical tests}

The fatigue tests were carried out on tubular samples of $316 \mathrm{~L}$ steel with average grain size 50 $\mu \mathrm{m}$, and with minimal preferred crystallographic orientation. With the microstructural information provided, the corresponding representative volume element (RVE) models of this steel based on both texture and morphology can be generated. Five different loading paths were considered at room temperature by Janssens [5], and the results are summarised in Fig. 1. It can be seen that fatigue life varies considerably with loading type and that significant scatter is observed within any one loading type. Loading histories were performed on a hydraulic, axialtorsional testing system, including fully reversed uniaxial loading (labelled A), fully reversed torsional loading $(\mathrm{T}), 90^{\circ}$ out-of-phase combined tension and torsion loading $(\mathrm{A}+\mathrm{T})$, a superposition of a larger-amplitude base uniaxial loading with a 10 times smaller-amplitude but 10 times higher-frequency uniaxial perturbed loading component $(A+A N)$, a superposition of a larger-amplitude base uniaxial loading with a 10 times smaller-amplitude but 10 times higher-frequency torsional perturbed loading component $(\mathrm{A}+\mathrm{TN})$. All the fatigue tests reported in this study are stress-controlled loading consisting of an $\mathrm{R}=-1$ sinusoidal loading curve (or a superposition of two sinusoidal loading curves) with a base frequency of $1 \mathrm{~Hz}$. The loading 
forms are detailed in Table 1. Examination of the 316L steel fatigue samples tested to failure demonstrated fatigue crack nucleation and ultimately failure within the gauge region of the tubular bar.
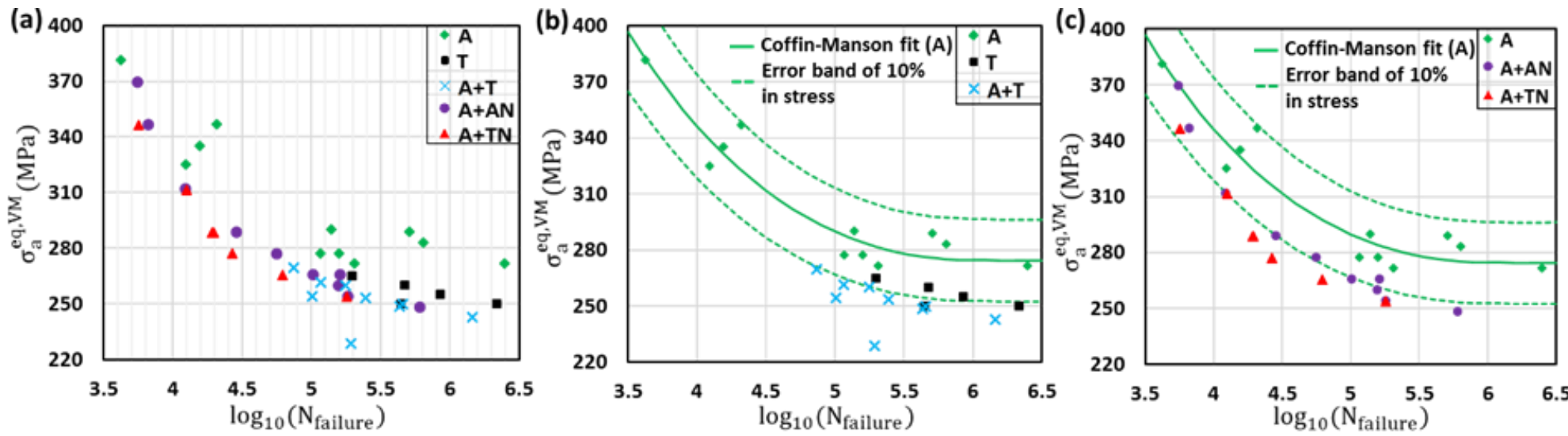

Fig 1. Fatigue lifetime data of $316 \mathrm{~L}$ steel at room temperature plotted using the von Mises equivalent stress amplitude under (a) all five loading cases, (b) pure axial (A), pure torsional (T) and axial+torsional $(\mathrm{A}+\mathrm{T})$ cases, (c) pure axial, axial+axial perturbed $(\mathrm{A}+\mathrm{AN})$ and axial+torsional perturbed $(\mathrm{A}+\mathrm{TN})$ cases

To compare the fatigue life obtained under different loading conditions, including uniaxial, torsional, $90^{\circ}$ out-of-phase non-proportional, and non-proportional perturbed, we use the equivalent von Mises stress $\sigma_{\mathrm{a}}^{\mathrm{eq}, \mathrm{VM}}$ to define the stress amplitude of any loading, and this is plotted as a function of the experimental observed fatigue cycles to failure across all tested samples, as shown in Fig. 1(a). Green diamonds indicate pure axial fatigue $\left(\sigma_{\mathrm{a}}^{\mathrm{eq}, \mathrm{VM}}=\sigma_{\mathrm{a}}\right)$, black squares indicate pure torsional fatigue tests $\left(\sigma_{\mathrm{a}}^{\text {eq,VM }}=\sqrt{3} \tau_{\mathrm{a}}\right)$, blue crosses represent $90^{\circ}$ out-of-phase non-proportional loading histories $\left(\sigma_{\mathrm{a}}^{\mathrm{eq}, \mathrm{VM}}=\sqrt{\sigma_{\mathrm{a}}{ }^{2}+\left(\sqrt{3} \tau_{\mathrm{a}}\right)^{2}}\right)$, purple circles and red triangles show axial base loading with axial perturbation and axial base loading with torsional perturbation data points respectively. To facilitate comparison among differing loading histories, the data points in Fig. 1(a) are separated into two groupings, which have been plotted in Fig. 1(b) and Fig. 1(c) respectively. Green trend lines for the purely uniaxial fatigue behaviour, including range of scatter, are included in all graphs for comparison. The uniaxial lifetime (green solid line) is plotted using the Coffin-Manson equation (11), which is often utilised for low cycle fatigue conditions, in which $\mathrm{N}_{\mathrm{f}}$ is cycles to failure.

$$
\sigma_{\mathrm{a}}^{\mathrm{eq}, \mathrm{VM}}=99\left(\mathrm{~N}_{\mathrm{f}}\right)^{0.05798}+1957\left(\mathrm{~N}_{\mathrm{f}}\right)^{-0.2608}
$$

The green dashed lines indicate a scatter-band of $10 \%$ in stress based on the fitted uniaxial lifetime curve. Evidently from Fig. 1(b), 316L stainless steel exhibits significant life scatter for the same stress ratio $(\mathrm{R}=-1)$ and stress magnitude. Significant scatter exists even for the case of pure uniaxial loading; for example, a maximum applied stress of $271 \mathrm{MPa}$ leads to lives from $10^{5.3}$ to $10^{6.4}$ cycles, demonstrating large variation. Similarly, the observed fatigue lifetime for pure torsional loading and $90^{\circ}$ out-of-phase non-proportional tension-torsion loading also reveal scatter. Given this, it is difficult to argue that there is significant difference observed between uniaxial and torsional loading fatigue life, indicated by the corresponding 
trend lines in Fig. 1(b), although the torsional behaviour tends towards the lower stress bound for the uniaxial behaviour. However, the $90^{\circ}$ out-of-phase non-proportional tension-torsion $(\mathrm{A}+\mathrm{T})$ data points are below the lower stress bound for the pure tension fatigue behaviour at low applied stress amplitude (no data are available for high applied stress), which is clearly differentiated and leads to a reduction of life compared to tension and torsion respectively as is clearly observed in Figs. 1(a) and (b).

Table 1. Five different cyclic loads and boundary conditions applied in experiment and in the RVE

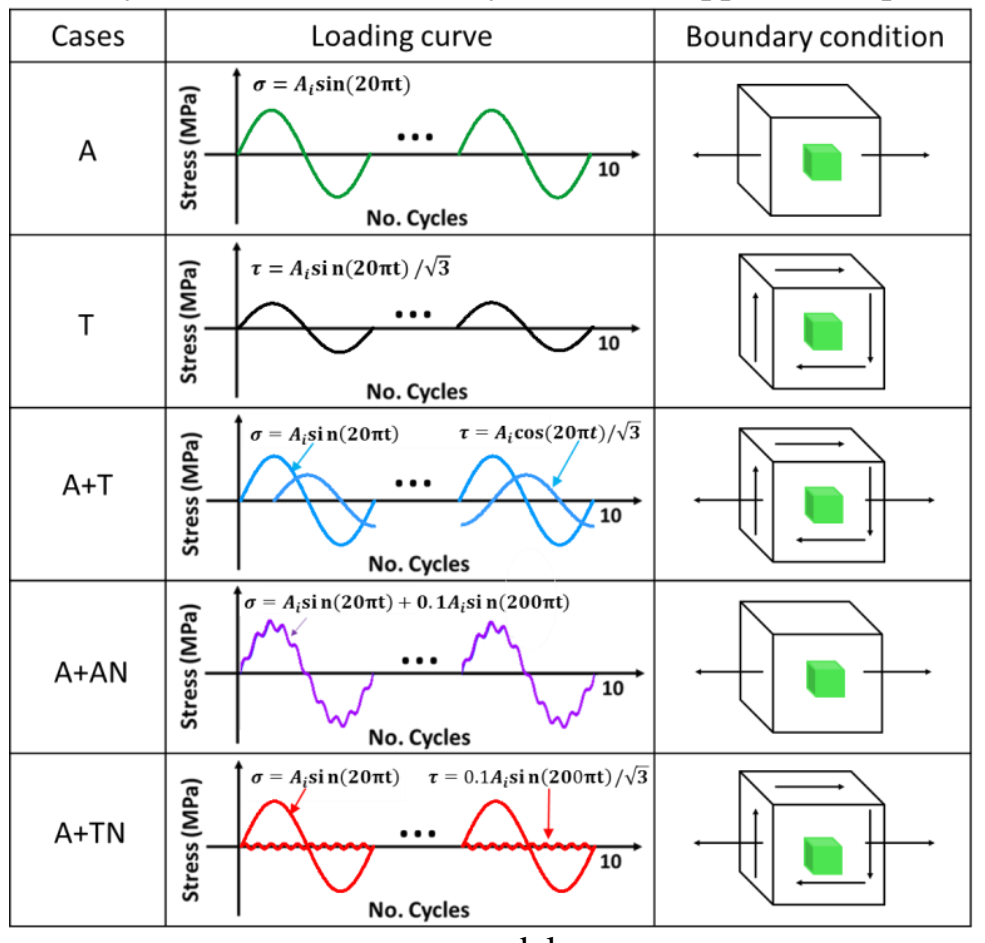

model

For the axial-torsional fatigue experiments involving a base uniaxial loading cycle and a nonproportional axial or torsional perturbation loading component, the fatigue data points are compared to pure uniaxial data, as shown in Fig. 1(c). From the observations, both axial and torsional fatigue perturbation tend to reduce the lifetime of the tested samples when compared to a lifetime curve based on fully reversed, stress-controlled uniaxial loading cycles. In addition, it is noted that the lifetime is reduced slightly more under the condition of torsional fatigue perturbation than that of axial perturbation, although the difference is small. More noticeable is that the reduction in life is much more apparent at low applied stress amplitude than for high stress, and for the latter, the inclusion of the perturbed non-proportional loading diminishes away to near zero compared to pure uniaxial loading.

\subsection{Crystal plasticity modelling}

To capture and explain the experimentally observed fatigue life variations and scatter under different loading conditions of 316L stainless steel, we use crystal plasticity modelling together with a fatigue crack nucleation criterion based on elastic stored energy density to simulate the detailed fatigue specimen microstructure. In this paper, a 3-dimensional representative grain geometry and morphology that is similar to the experimental characterized samples is generated. The 3D RVE model is then meshed in Abaqus CAE. A user defined material UMAT subroutine incorporating crystal plasticity is then used for analysing the fatigue behaviour at the grain length scale. Three-dimensional controlled Poisson voronoi tessellation has been 
utilized to generate the representative microstructural model in this study. The mean grain size and Gaussian grain size distribution function were chosen to represent the experimental microstructures. Experimental characterisation, using the software (ImageJ) analysis, gave the minimum, mean and maximum grain size within this material to be $\sim 10 \mu \mathrm{m}, \sim 50 \mu \mathrm{m}$, $\sim 120 \mu \mathrm{m}$ respectively. Therefore, the grain size control parameter here was set to give a distribution of normalised grain sizes as shown in Fig. 2(a). The texture in the investigated 316L stainless steel samples has been utilized to construct the RVE model in Fig. 2. The representative polycrystalline model has been shown to reproduce the average stress-strain response at different strain rates at a temperature of $20^{\circ}$ (see below). In this study, several differing morphological representations have been developed, whilst maintaining the same crystallographic orientations, in order to assess their role in predicted stored energy and hence fatigue life.

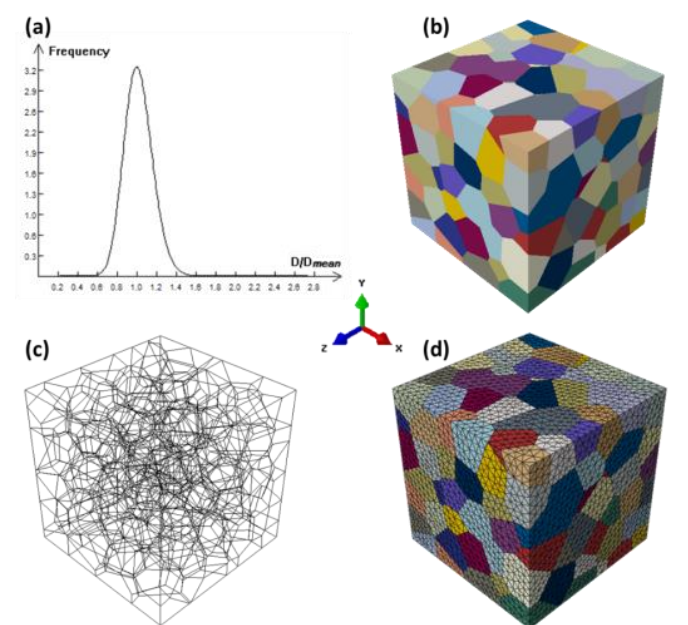

Fig 2. Crystal plasticity finite element model illustrating (a) distribution of grains against normalized grain size (b) surface grain morphology (c) 3D network of internal and external grain boundaries (d) the tetrahedral element meshed model

The controlled Poisson voronoi tessellation model was imported into ABAQUS CAE to mesh. This model includes 216 surface grains, as shown in Fig. 2(b). An extremely complex grain boundary network within this 3D polycrystalline model resulted, illustrated in Fig. 2(c). 171,947 ten-noded tetrahedral elements (C3D10) with an average element size of $\sim 13 \mu \mathrm{m}$ were employed in the model shown in Fig. 2(d). The CPFE modelling methodology with the fatigue crack nucleation criterion based on elastic stored energy density has been described in section 2. In this study of $316 \mathrm{~L}$, the development of SSD density is considered to be significantly more substantial than that for GND accumulation due to the large grained steel microstructures investigated. Therefore, it is argued that the GND density is not likely to be as important as the SSD accumulation and consequently, the localized development of stored energy density is dominated by SSDs over that of GNDs. The uniaxial stress-strain response of the $316 \mathrm{~L}$ steel is used in order to calibrate the crystal plasticity model described above. Uniaxial strain controlled loadings with different strain rates have been applied up to 5\% strain. The slip related parameters and hardening coefficient $\lambda$ in the crystal plasticity model have been identified and determined by ensuring the bulk uniaxial hardening response of FCC polycrystalline austenitic steel is appropriately captured by the model. The properties so determined are listed in Table 2. The computed crystal plasticity stress-strain responses at different strain rates obtained from the model are shown in Fig. 3, giving reasonably good agreement with experimental data. 
Table 2. Materials properties for crystal slip in $316 \mathrm{~L}$ steel

\begin{tabular}{c|c|c|c|c|c|c|c}
\hline$\tau_{\mathrm{co}}(\mathrm{MPa})$ & $\mathrm{b}(\mu \mathrm{m})$ & $\lambda\left(\mu \mathrm{m}^{-2}\right)$ & $v\left(\mathrm{~s}^{-1}\right)$ & $\Delta \mathrm{F}(\mathrm{J} / \mathrm{atom})$ & $\rho_{\mathrm{ssdm}}\left(\mu \mathrm{m}^{-2}\right)$ & $\mathrm{k}(\mathrm{J} / \mathrm{K})$ & $\Delta \mathrm{V}\left(\mu \mathrm{m}^{3}\right)$ \\
\hline 97 & $2.5 \times 10^{-4}$ & 175 & $1 \times 10^{11}$ & $2.6 \times 10^{-20}$ & 0.01 & $1.38 \times 10^{-23}$ & $39.37 \mathrm{~b}^{3}$ \\
\hline
\end{tabular}

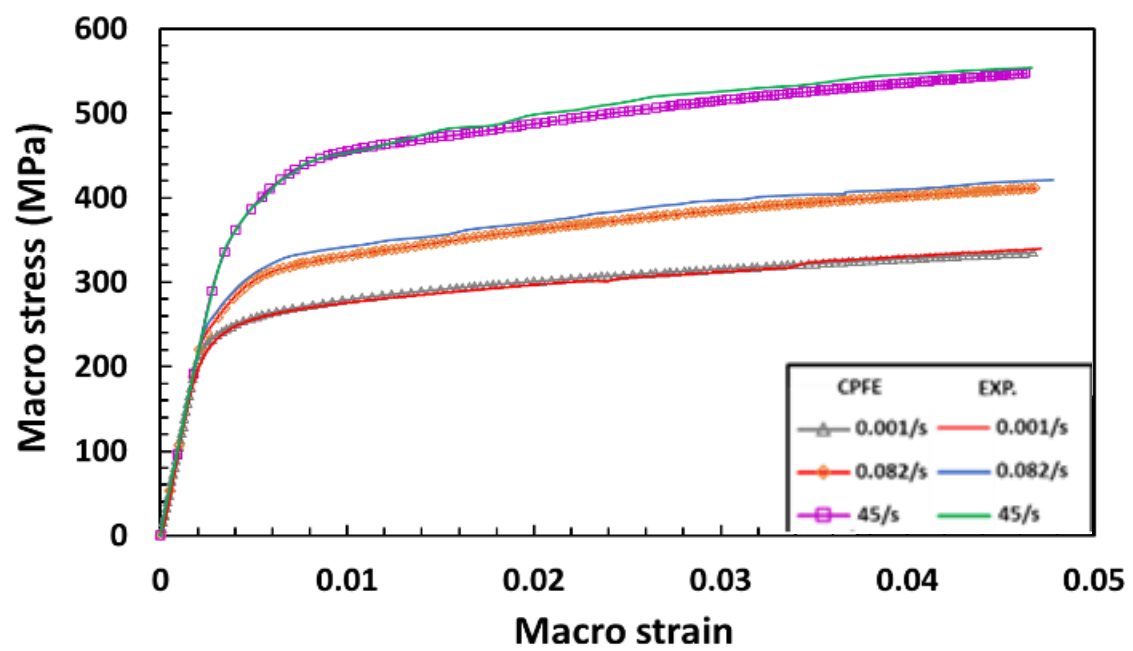

Fig 3. Calibrated CPFEM response against experimental macroscopic stress strain data for $316 \mathrm{~L}$ at room temperature

The experimental fatigue loading conditions described above and shown schematically in Table 1 are imposed on the model RVE by firstly encapsulating the RVE into a homogeneous medium as shown in Fig. 4. The experimental loading conditions are imposed on the boundaries of the model shown in Fig. 4(a) and thus indirectly transmitted to the crystal plasticity RVE region. For the latter, the grains are modelled using elastically anisotropic crystal plasticity with random crystallographic orientations. An X-plane cut view of the model is shown in Fig. 4(b) with a magnified map of the central surface grain morphologies exhibited in Fig. 4(c). 

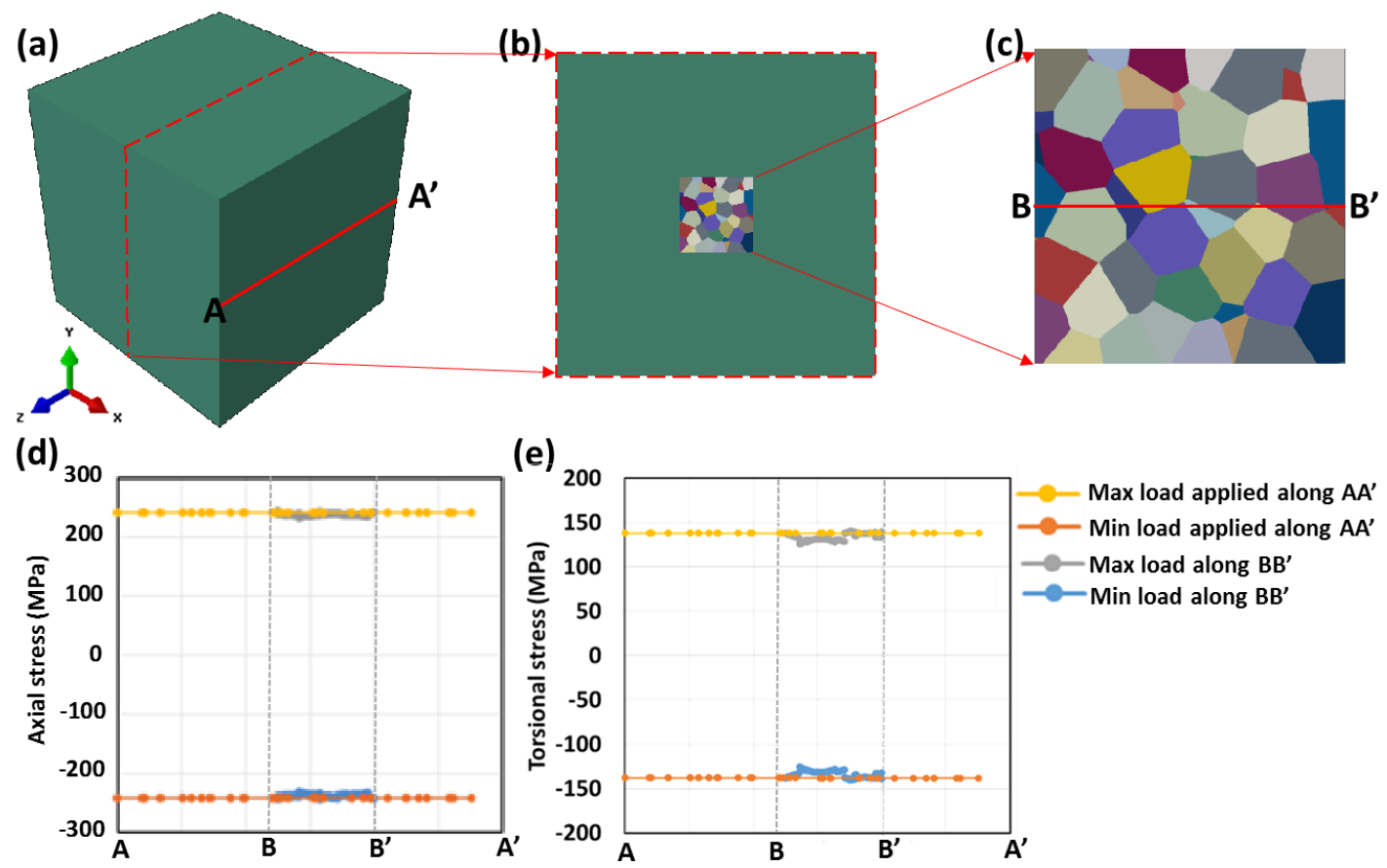

Fig 4. (a) Isotropic steel region encapsulating the representative polycrystalline model; (b) an X-plane cut view which has been highlighted in (a); (c) close-up grain map of region marked in (b); the applied maximum and minimum load on the outer surface along AA' (highlighted in (a)) for pure axial and pure torsional specimens with the max von Mises equivalent stress of 240MPa have been shown in (d) and (e) respectively, and the corresponding stress amplitude on the inner RVE surface have been plotted along BB' (highlighted in (c)).

The determination of the appropriate boundary and loading conditions for the CP model is necessary in order to obtain the correct mechanical and physical behaviour developed in the experiments. Therefore, a path has been defined on the outer surface of the large steel matrix and inner surface of the CP model respectively, that is, AA' (marked in Fig. 4(a)) and BB' (marked in Fig. 4(c)), in order to ensure that the appropriate loading is applied to the polycrystalline model. Evidently from Fig. 4(d), under stress-controlled pure uniaxial conditions, the applied maximum and minimum load along AA' is almost identical to the stress generated on the inner RVE surface along BB', although there are small fluctuations ( $10 \mathrm{MPa})$ observed. Similarly, a reasonably good representation has been obtained between the load applied on the outer surface and the load induced on the inner polycrystal surface under the condition of pure torsion, as shown in Fig. 4(e). The influence of the observed stress fluctuations in both cases are anticipated to be very small with respect to the load applied. In addition, the dimensions of the RVE model are sufficiently small compared to the tubular sample and applied loading that it becomes very reasonable to argue that locally, the loading is uniform. In this way, the applied loading paths for pure tension, pure torsion, $90^{\circ}$ out-ofphase tension and torsion, tension with tension perturbation and tension with torsion perturbation listed in Table 1 have been developed.

\subsection{Stored energy and slip accumulation in proportional and non-proportional loading}

In this study, we focus only on two key quantities for analysing the fatigue behaviour of the tested 316L stainless steel, namely (i) cyclic effective plastic strain rate $\dot{\mathrm{p}}$ (i.e. plastic strain per 
cycle) at steady state, and (ii) cyclic stored energy density rate $\dot{G}$ which is defined by Eq. (8). $\dot{p}$ and $\dot{G}$ are assessed for their ability to differentiate and explain the experimentally observed fatigue failures. In addition, the overall distributions of the accumulated slip and stored energy density over a number of cycles are also investigated in order to explain the experimental observations.

The modelled fatigue specimens are subjected to 10 cycles of $\mathrm{R}=-1$ stress-controlled loading. It is found from earlier work $[29,31]$ that even a relatively small number of cyclic loadings may be sufficient to allow the local spatial development of dislocation densities, the networks of stresses and accumulated slip in the microstructure to be established. While the magnitudes of the quantities continue to evolve with cycling, the spatial distributions tend not to change with subsequent cycles. In other words, the evolutions of slip, stress and dislocation fields tend to be stable over a certain number of cyclic loadings and then plastic shakedown may occur to enable sensible extrapolations to be made for subsequent fatigue cycles. In order to facilitate comparison, the five load cases have been separated into two groupings in the subsequent analysis; that is, one grouping contains assessment of pure tension, pure torsion, out-of-phase non-proportional tension and torsion, and the other pure tension, pure tension with nonproportional tensile perturbation, and pure tension with non-proportional torsion perturbation.

The local stored energy per cycle (Eq. (8)) at the highest hotspots is assessed for all samples across all loading paths, as shown in Fig. 5(a) and (b). For each loading case, the cyclic stored energy becomes larger with higher applied stress. From Fig. 5(a), the model predictions suggest significant difference in energy stored per cycle for non-proportional $(A+T)$ and pure axial/pure torsional loading, with significantly higher cyclic stored energy for the non-proportional loading, indicating shorter fatigue life, which is consistent with experiment observations in Fig. 1(b). In addition, a slightly higher cyclic stored energy is predicted for pure axial loading compared to pure torsion, suggesting that the torsional loading should give rise to longer experimental fatigue lives. However, Fig. 1(b) shows that the torsional fatigue lives lie at the lower range of the uniaxial lives for given stress.

Further, it is observed from Fig. 5(b) that the CPFE modelling predicts cyclic stored energy $\dot{\mathrm{G}}$ to be slightly higher for the perturbed fatigue cases than for the pure uniaxial loading, for all applied stress levels. Although the difference is small, non-proportional torsional perturbation superposed on axial fatigue gives higher $\dot{G}$ than that for axial perturbed fatigue. This observation indicates that the fatigue life is anticipated to be reduced more by torsional perturbation than axial perturbation superposed on the axial fatigue loading. This behaviour is shown by the experimental data in Fig. 1(c). Hence the cyclic stored energies calculated are consistent with experimental (proportional and non-proportional) fatigue life measurements with the possible exception of the torsional loading (but see later). 

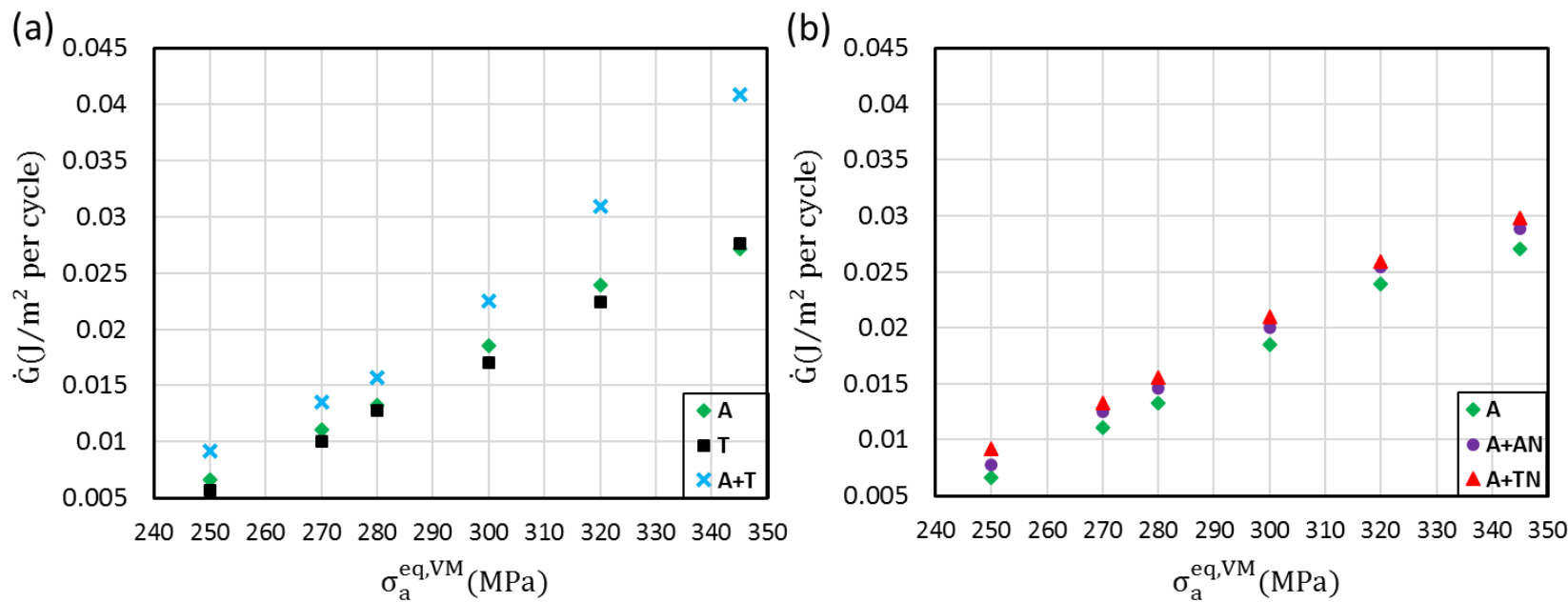

Figure 5. Calculated stored energy density rate against experimental applied stress plotted using the von Mises equivalent stress amplitude for 316L steel (a) pure axial, pure torsional and axial + torsional loads (b) pure axial, axial+ axial perturbation and axial + torsional perturbation

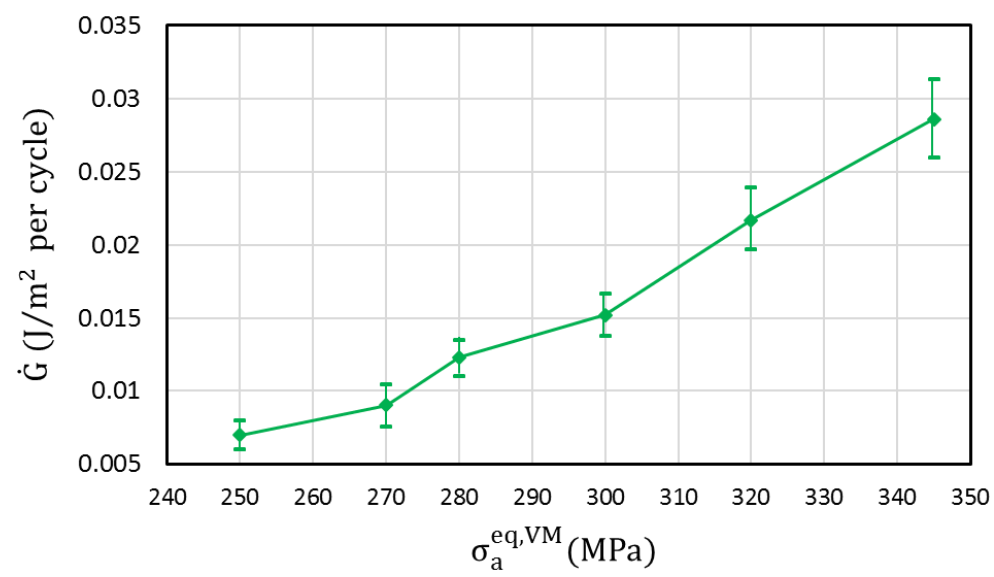

Figure 6. Calculated stored energy density rate vs stress applied (von Mises equivalent stress amplitude) under pure axial loading conditions for five different microstructures but with the same texture

Considerable differences in cyclic stored energy arise from the differing fatigue loading types in Fig. 5, but in addition, quite considerable fatigue life scatter is observed for pure uniaxial loading in Fig. 1(a), giving scatter bounds of $+/-10 \%$. It is useful, therefore, to assess the range in magnitude of cyclic stored energies arising from random microstructural variation versus that arising from differences in loading type in order both to interpret the significance of stored energy magnitude with loading change and to explain fatigue scatter arising for the same loading type. In order to do this, five different sets of microstructure realisations (grain morphology) but maintaining the same random texture have been analysed under pure uniaxial fatigue loading over 10 cycles (as before) with stress ratio of $\mathrm{R}=-1$ and peak Mises stresses of $250,270,280,300,320$ and $345 \mathrm{MPa}$ respectively. For each applied stress, the average of the five peak values of cyclic stored energy $\dot{G}$ obtained has been determined and plotted in Fig. 6 . In addition, the range of peak energy over the five realisations, resulting solely from microstructure morphology variation, is also shown by the error bars for each stress. Firstly, Fig. 6 shows that the variation of cyclic stored energy at a given stress resulting from microstructural differences alone is small compared to the differences in stored energy arising 
from proportional versus non-proportional loading in Fig. 5(a). Hence the differences in stored energy shown in Fig. 5(a) cannot arise from morphological arrangements alone; they are significant and originate from load path change. Second, the variations in cyclic stored energy for given stress in Fig. 6 which do result from morphology change reflect the fatigue scatter that would be anticipated in repeating the same kind of test. The variation in cyclic stored energy is about $10 \%$ with morphology variation, and if we simplify to assume steady state (plastic shakedown) conditions have been achieved, then eqn. (8) indicates we would anticipate a range of scatter on life of about $10 \%$. This isn't far off what is experimentally observed for pure uniaxial fatigue in Fig. 1(b).

An analysis has been carried out for the inverse cyclic stored energy rate, $\dot{\mathrm{G}}^{-1}$, which is related to fatigue cycles to failure, as described by eqn. (10). The relationship becomes clear if, for sake of explanation, we assume a constant cyclic energy rate, $\dot{G}$, in eqn. (10) giving $G_{c}=\dot{G} N_{f}$ such that $G_{c} \dot{G}^{-1}=N_{f}$ in which $G_{c}$ is constant. Fig. 7(a) and (b) show clear differentiation of the non-proportional $(\mathrm{A}+\mathrm{T})$ loading giving significantly shorter fatigue lives than those for proportional tension and torsion, again in agreement with experimental observations in Fig. 1(b). In addition, Fig. 7(b) indicates that at stresses $<280 \mathrm{MPa}$, pure torsional fatigue gives rise to higher fatigue cycles to failure than pure uniaxial fatigue. However, $\dot{G}^{-1}$ for pure tension and pure torsion tend to converge for stresses above $\sim 280 \mathrm{MPa}$, suggesting fatigue lifetimes which also converge. The experimental data in Fig. 1(b) show shorter fatigue lives in pure torsion than in pure uniaxial loading for stresses between 250 to $270 \mathrm{MPa}$ but lifetimes converge at about $280 \mathrm{MPa}$. Fig. 7(c) shows that the superposition of non-proportional axial or torsional perturbation leads to a fatigue lifetime reduction, especially at low stress, which diminishes away for stresses above about $300 \mathrm{MPa}$. This behaviour is very nicely observed in the experimental data shown in Fig. 1(c).
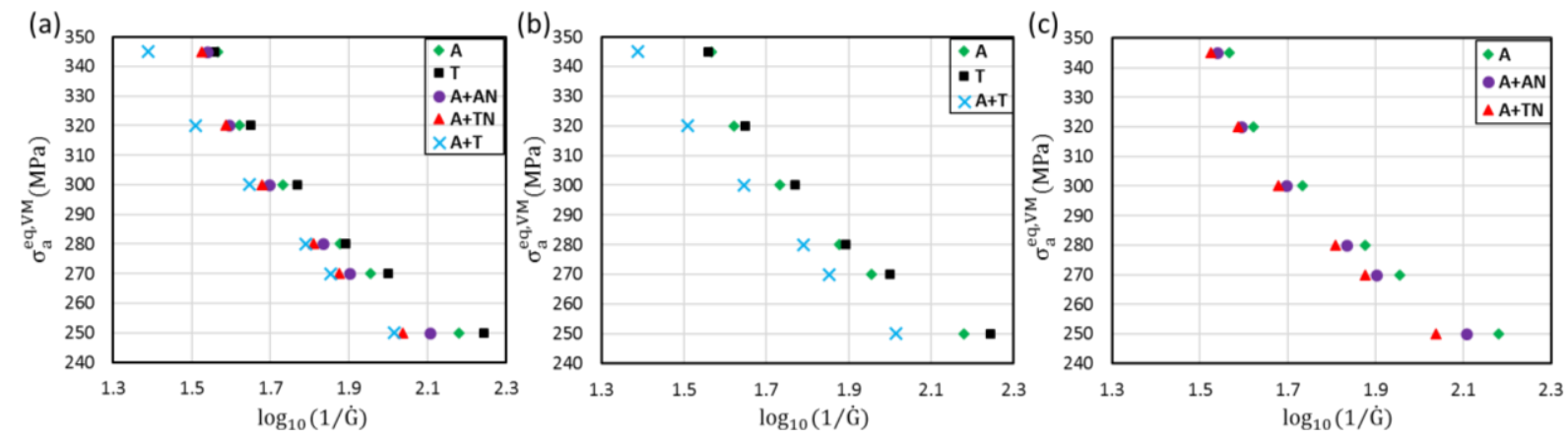

Figure 7. Applied Mises stress amplitude vs the predicted number of cycles per unit energy stored at the highest stored energy density hotspots for (a) all samples (b) samples which are subjected to pure axial, pure torsional and non-proportional axial and torsional loading (c) samples which are subjected to pure axial, axial with axial perturbation and axial with torsional perturbation respectively

Significant heterogeneity is observed together with strong localization of accumulated strain across the microstructure and within individual grains for each of five investigated loading paths, so a more quantitative assessment of the frequency distribution of plastic strain accumulation for maximum applied Mises stresses of 250, 300 and $345 \mathrm{MPa}$ after ten loading cycles is presented in Fig. 8(a)-(e). For all loading paths, the range of plastic strain observed increases with applied stress. In particular, for the same applied stress magnitude, the nonproportional $\mathrm{A}+\mathrm{T}$ loading generally generates a wider range of strain activation than that for 
the other loading cases. This loading leads to shorter experimental fatigue lives than for axial or torsional fatigue because of the resulting higher stored energies. The torsional loading shows a slightly broader range of strain activity than that for pure axial, particularly for higher stresses.
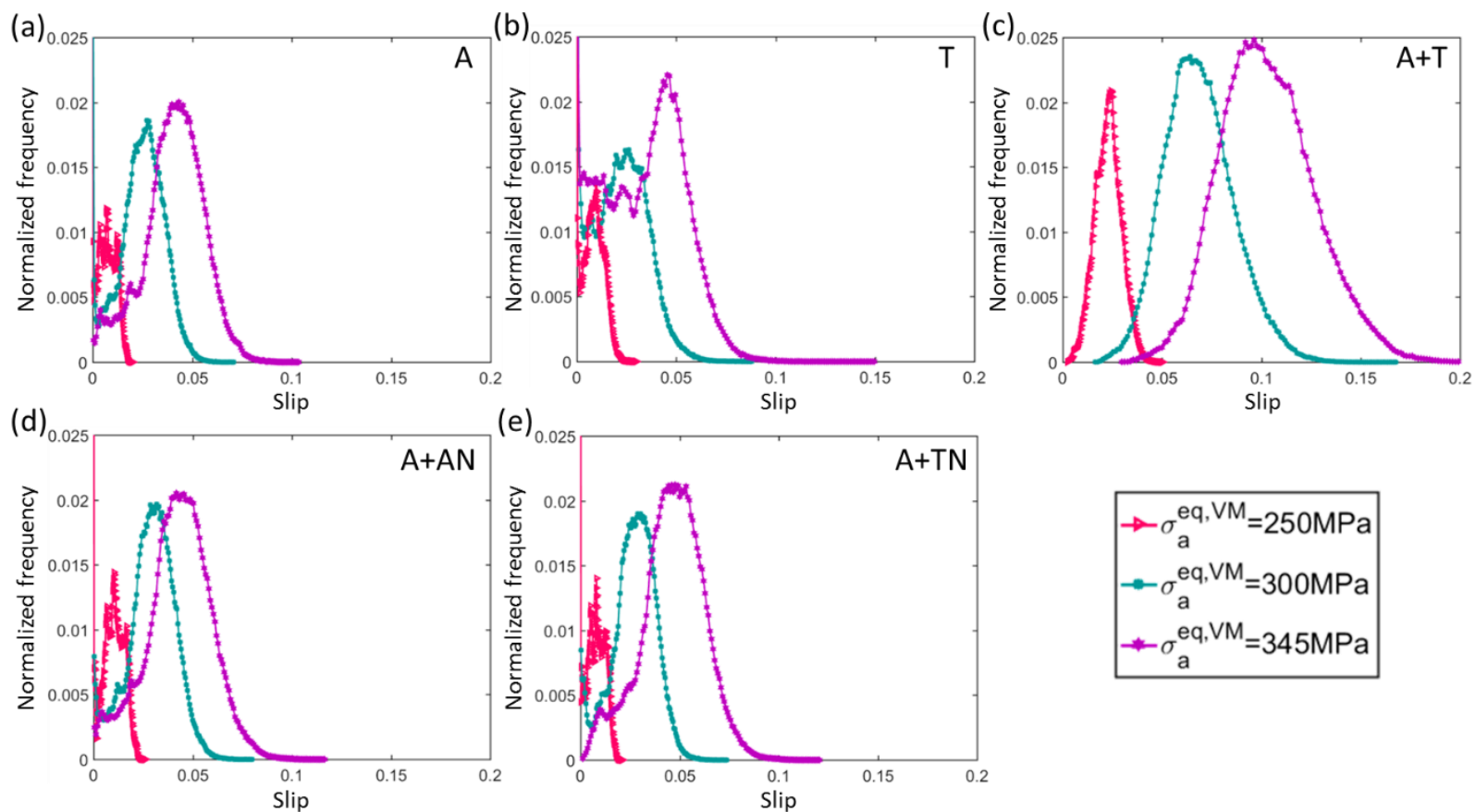

Fig. 8 Normalized frequency distribution plots of accumulated slip after ten fatigue cycles for $\mathrm{R}=-1$ with max Mises stress of $250 \mathrm{MPa}, 300 \mathrm{MPa}$ and $345 \mathrm{MPa}$ respectively for the five loading types shown

\subsection{Predicted multiaxial and non-proportional fatigue life}

The stored energy density rates described above facilitate the ranking of the observed fatigue behaviour of the tested stainless steel specimens with higher $\dot{G}$ indicating shorter fatigue life, as demonstrated in Fig. 7. A more quantitative assessment, however, is possible since it is argued that fatigue crack nucleation occurs once the local stored energy $\mathrm{G}$, which accumulates over fatigue cycles, achieves the critical value, $\mathrm{G}_{\mathrm{c}}$ sufficient to generate new surfaces with surface energy equating to $G_{c}$. The evolving stored energy, $G$, is calculated using Eqns. (8) and (10) within the CP model during cyclic loading. It only remains to determine the critical stored energy $\mathrm{G}_{\mathrm{c}}$ and this is obtained from consideration of the axial fatigue data alone. To do this, the cyclic rate of change of $G$ is obtained from the model at the end of ten cycles at which point it is reasonably assumed that it has stabilised and has ceased to change in subsequent cycles. Hence utilising Eq. (10) in combination with the experimental axial fatigue life data in Fig. 1(a) enables the critical stored energy to be determined as $591.7 \mathrm{~J} / \mathrm{m}^{2}$. We note that the cycles to crack nucleation are here equated to the experimentally observed cycles to failure, but the fatigue lives for most of the tested samples are high and close to the high cycle fatigue regime in which fatigue crack nucleation dominates and accounts for most of fatigue life.

With the critical stored energy established for the $316 \mathrm{~L}$ steel, it then becomes possible to predict all the fatigue lives for the torsional ( $\mathrm{T})$, axial plus torsional $(\mathrm{A}+\mathrm{T})$, axial plus axial perturbation $(\mathrm{A}+\mathrm{AN})$ and axial plus torsional perturbation $(\mathrm{A}+\mathrm{TN})$ fatigue tests. The predicted fatigue life results obtained together with the experimental data are shown in Fig. 9. Here, as before, the symbols correspond to experimental data and lines to model predictions. The green shaded 
region corresponds to the $10 \%$ range on the axial fatigue only. Reasonable agreement is obtained for the axial fatigue (given these experimental data were used to extract the critical stored energy), though we note for subsequent discussion that the fatigue crack nucleation criterion requires only a single material quantity; the critical stored energy $G_{c}$. Moving to the non-proportional axial plus torsional loading shown in Fig. 9(a), the significantly shorter lives observed for the latter are predicted by the stored energy. The predicted fatigue life for pure torsion is marginally longer than that for pure tension for low and high applied stresses ( $<280 \mathrm{MPa}$ and $>345 \mathrm{MPa}$ ), but marginally shorter for mid-range stresses (280-345 MPa). The CP model based on stored energy differentiates appropriately most of the loading types and their respective fatigue life rankings without any empiricism or fitting, therefore at least indicating that some of the important slip mechanisms contributing to the fatigue life differences are captured. Mises stress amplitude approaches to fatigue life would not, of course, show any difference in fatigue life originating from non-proportional loading carried out at the same Mises stress; the current stored energy approach captures this effect directly.

Fig. 9(b) shows predicted and experimental fatigue lives corresponding to the axial fatigue plus fatigue perturbation loading together with the pure axial data for comparison. The detrimental effect of the introduction of the non-proportional axial and torsional perturbation loading is captured by the stored energy, showing that for lower stresses, substantial decreases in fatigue life are observed and captured by the stored energy, but that for higher stress amplitude, the non-proportional effects diminish away. In addition, while the predicted differences are quite small, the stored energy also indicates that the superposition of torsional perturbation is more detrimental than that for axial fatigue perturbation. This behaviour is also very nicely observed in the experimental data shown in Fig. 9(b).
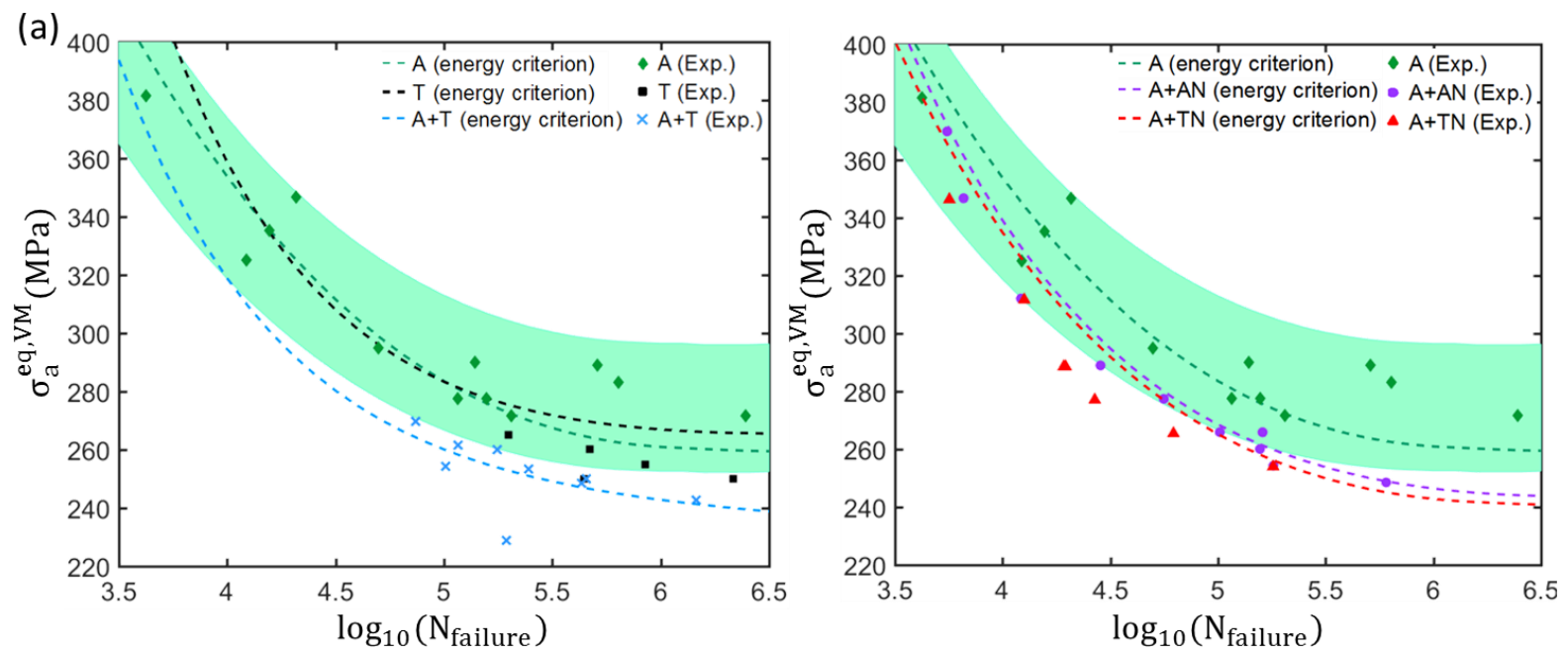

Fig 9. S-N diagrams for 316L stainless steel predicted from stored energy and observed experimentally for the range of proportional and non-proportional loading regimes and stress states shown. The critical stored energy is obtained from consideration of axial loading only. The shaded green region indicates $10 \%$ range on the axial fatigue data.

\section{Non-proportional fatigue analysis of fine-grained nickel-based superalloy RR1000}

The work presented in this section addresses the influence of five different stress-controlled loading paths on the fatigue life variations of Ni-based alloy RR1000. With knowledge of the experimental results provided by Rolls-Royce, a systematic CPFE modelling study is carried out to investigate the mechanistic basis of the fatigue lifetimes under differing loading paths. The nickel alloy considered is fine-grained, and as a consequence, the effects of GND density are anticipated to be more influential in the behaviour. 


\subsection{Mechanical tests}

A series of multi-axial load-controlled proportional and non-proportional fatigue tests were conducted by Rolls-Royce. Twenty-eight thin-walled hollow cylindrical test specimens were used in the fatigue test programme. Specifically, five different load paths were investigated to determine the uniaxial and multi-axial fatigue behaviour of fine-grained RR1000 at room temperature. Table 3 shows the applied tension-torsion stress paths and the corresponding loading histories for a complete fatigue cycle, where $\sigma_{x x}$ and $\tau_{x y}$ are the applied axial and shear stresses respectively. Three different proportional loading paths, including a pure uniaxial (A), pure torsion (T), and a proportional in-phase (IP) test, are studied. In addition, two nonproportional fatigue loading paths are studied, including a 'square' (SQ) cycle, and uniaxial followed by pure torsion cycle (AT). All the tests were carried out at stress ratio $R=-1$.

The test results are summarized in Fig. 10. The non-proportional tests (AT and SQ) give shorter fatigue lives for the same applied Mises stress amplitude than the equivalent proportional tests. The difference increases as the Mises stress range decreases. From the previous study on 316L steel, this was attributed to the larger number of slip systems activated during non-proportional loading. In addition, there is a clear separation of fatigue life for given applied stress range (< $\sim 0.9$ ) for pure uniaxial, pure torsion and in-phase tension-torsion tests, with highest fatigue lives attained for pure torsion (T), followed in order by proportional axial-torsion (IP), axial (A), and non-proportional axial-tension cycles (AP) and (SQ).

Table 3. Stress controlled fatigue loading histories for each of 5 cases in Ni alloy RR1000

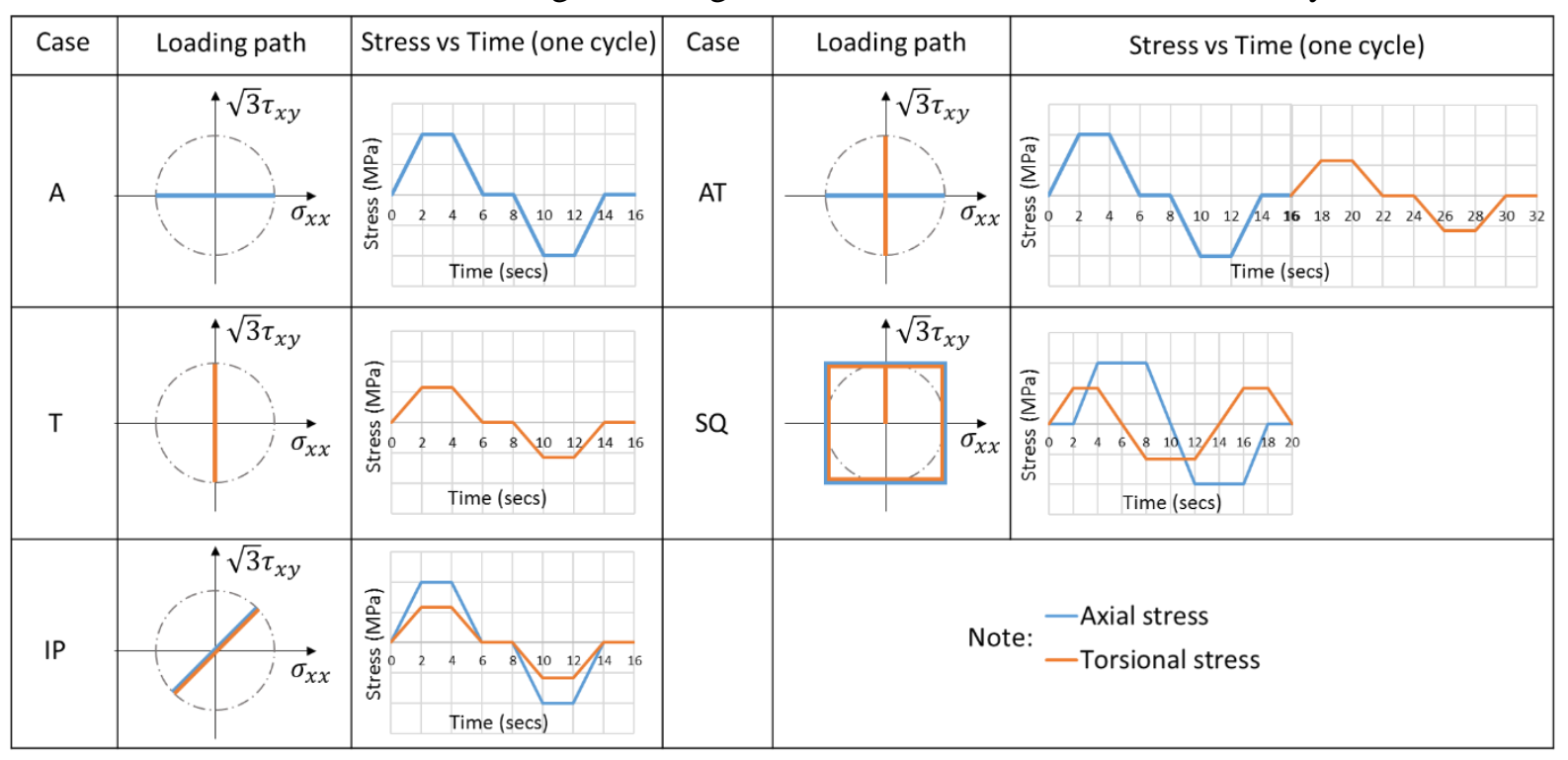


Evidently, non-proportional tension-torsion fatigue tests in RR1000 in Fig. 10 lead to a reduction of life compared to proportional loading, at the same Mises stress range. The fatigue life of in-phase tension-torsion is observed to lie between that of pure tension and pure torsion, but which is also stress dependent. These interesting features are investigated using the same crystal plasticity modelling methodology described earlier together with the fatigue crack nucleation criterion based on elastic stored energy density. In this instance, because the material is fine-grained, the role of geometrically necessary dislocations is thought to be important particularly for the stored energy density and so GND evolution is explicitly included and investigated.

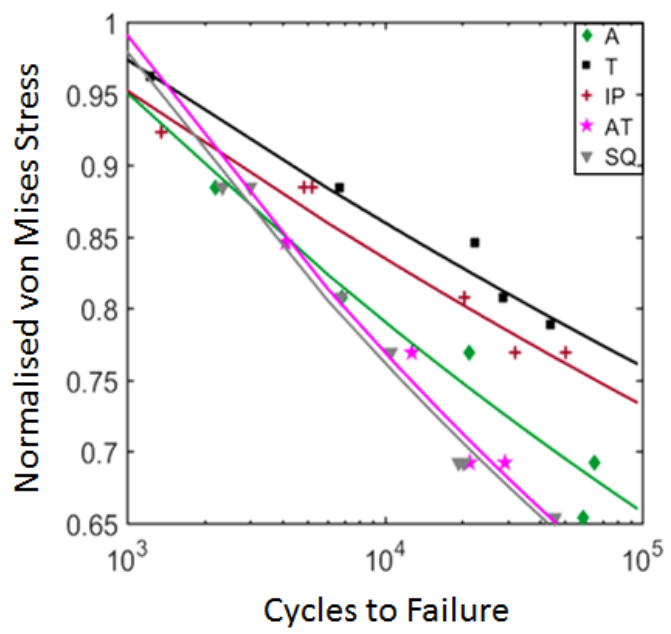

Figure 10. Normalized effective (von-Mises) stress against life to failure from multi-axial fatigue tests. Lines indicate trend only.

\subsection{Crystal plasticity RVE model}

A 3D digital microstructure which reproduces the statistics of RR1000 grain size and texture has been generated using commercial 3D microstructure construction software DREAM3D

(a)

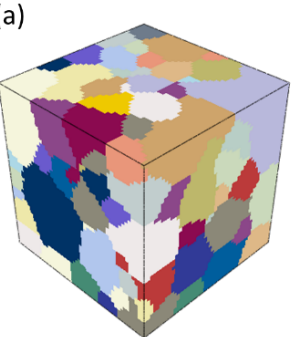

(c)

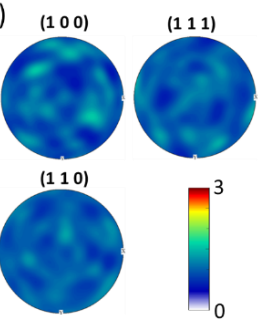

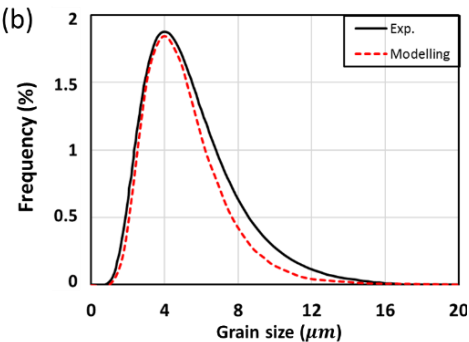

(d)

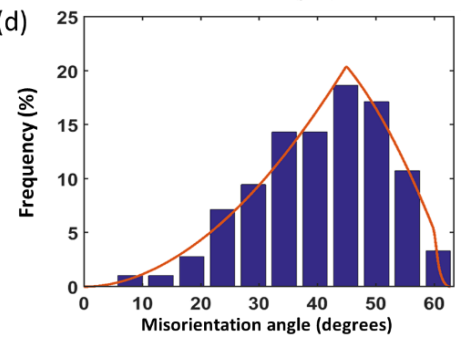

Figure 11. (a) Synthetic microstructure generated by Dream3D [32], (b) the grain size distribution and (c) pole figures and (d) misorientation distribution of microstructure shown in (a) 
[32], which contains synthetic instantiation optimization techniques. Hence, a representative computationally comparable model of RR1000, consisting of 168 surface grains and 640003 dimensional 20-noded elements with reduced integration (C3D20R) is generated, shown in Fig. 11 , in order to perform detailed fatigue lifing studies. Material properties for fine-grained nickel alloy RR1000 are summarized in Table 4.

Table 4. Material properties for crystal slip in PM Ni polycrystalline RR1000

\begin{tabular}{ccccccccc}
\hline$\tau_{\mathrm{c} 0}(\mathrm{MPa})$ & $\mathrm{b}(\mu \mathrm{m})$ & $\lambda\left(\mu \mathrm{m}^{-2}\right)$ & $v\left(\mathrm{~s}^{-1}\right)$ & $\Delta \mathrm{F}(\mathrm{J} /$ atom $)$ & $\rho_{\mathrm{ssdm}\left(\mu \mathrm{m}^{-2}\right)}$ & $\mathrm{k}\left(\mathrm{JK}^{-1}\right)$ & $\mathrm{E}(\mathrm{MPa})$ & $\mathrm{G}_{12}(\mathrm{MPa})$ \\
450 & $3.5072 \mathrm{E}-4$ & 150 & $1.0 \mathrm{e}+11$ & $3.456 \mathrm{E}-20$ & 0.05 & $1.381 \mathrm{e}-23$ & $210 \mathrm{E}+3$ & $90 \mathrm{E}+3$ \\
\hline
\end{tabular}

\subsection{Model fatigue behaviour of RR1000}

An assessment has been carried out of the GND density evolution at predicted hotspots which result from the five proportional and non-proportional loadings in order to investigate whether this quantity may be identified to be associated with the measured fatigue life to failure under each loading path. The corresponding GND densities and their evolutions over 10 fatigue cycles at the locations of highest GND hotspots are extracted from the full field crystal plasticity calculations and plotted in Fig. 12. Here it is observed that the GND density rapidly increases over the first few cycles in all cases followed by stabilisation. It is also interestingly observed that the GND density evolution under non-proportional square loading (SQ) is rather different (as is that for non-proportional loading AT, but to lesser extent) to the other three loading cases, showing a higher progressive cyclic increase after saturation than for the other proportional loadings.

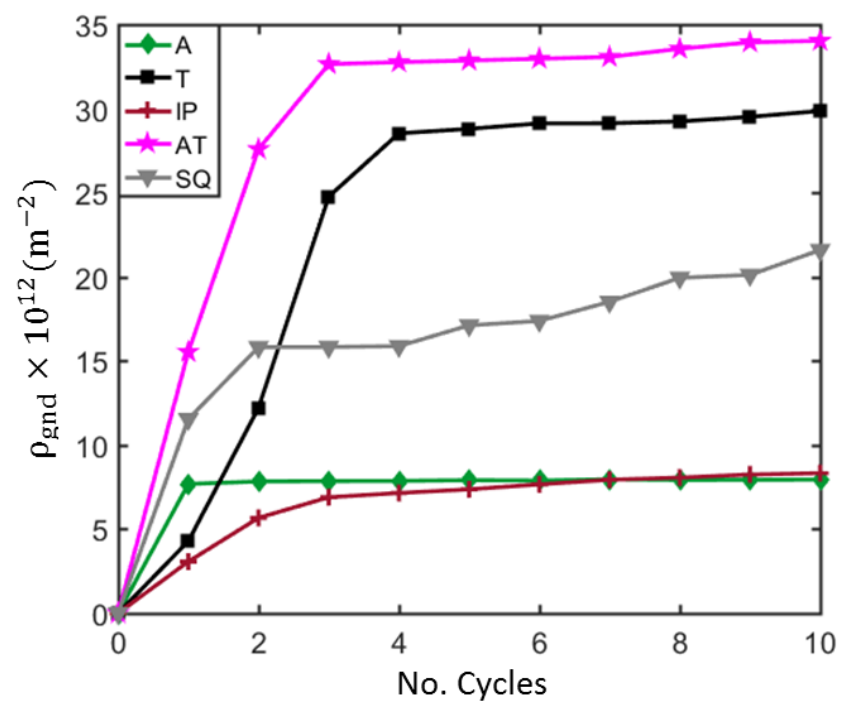

Figure 12. GND density change at the location of highest GND density for each loading case over 10 fatigue cycles 
In terms of the absolute value of the GND density at 10 cycles, loading AT exhibits the highest GND density $\left(\sim 3.4 \times 10^{13} \mathrm{~m}^{-2}\right)$ and the uniaxial loading (A) the least $\left(\sim 7.5 \times 10^{12} \mathrm{~m}^{-2}\right)$, developed over 10 fatigue cycles. Therefore, this quantity (at 10 cycles) does not correlate directly with fatigue life. However, the rate of increase of GND density is more interesting, and indicative of behaviour for later fatigue cycles, since local, high GND density drives up local stress and hence local stored energy, as reflected in Eqn. (8). The cyclic stored energy rates are therefore determined for the cases where GND density evolves and when it is maintained at zero. These results are shown in Figure 13(a) for a normalised applied stress range of 0.8 in which it is seen that the cyclic stored energy rate ranks from highest to lowest in a way that matches exactly the fatigue lifetime observations (see Figure 10) from shortest to longest when the GND density evolution is included in the calculation. When it is switched off in the calculations, the peak cyclic stored energy ranking no longer matches the experimental observations, as shown in Figure 13(a). In fact, without recognition of the influence of GND density on stored energy, the predicted fatigue ranking is completely wrong. It is argued that in fine-grained RR1000, GND density plays a significant role in generating a contribution to local elastic energy. An important aspect seems to be the rate at which the GND density evolves cyclically. Recall Fig. 12 shows the highest rates of evolution of GND density occur for the two non-proportional loading cases. Is GND density evolution, therefore, the key mechanistic difference in fatigue life between proportional and non-proportional loading, and is it influenced by the activation of more slip systems in non-proportional loading? This question is assessed further in the discussion.

(a)

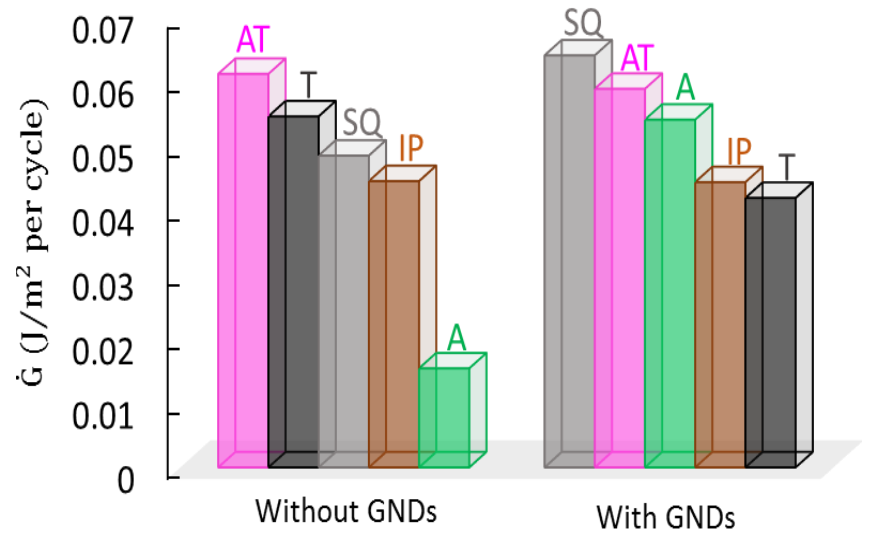

(b)

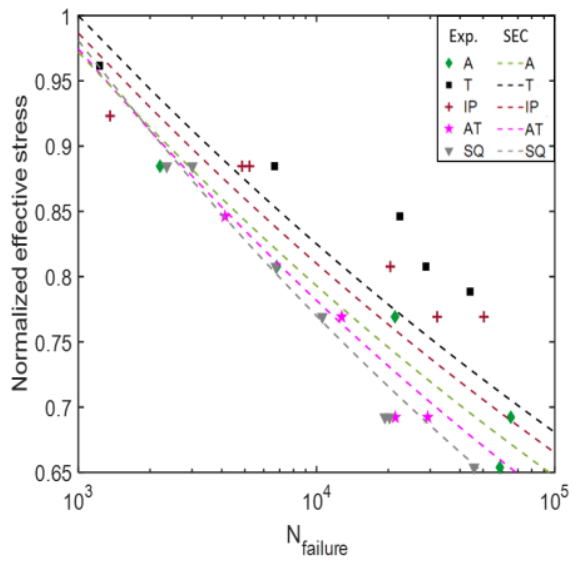

Figure 13. (a) Computed cyclic stored energy density without GNDs and with GNDs under differing loading paths at the given applied normalized stress range (0.8), and (b) Fatigue lifetimes for RR1000 predicted from stored energy and observed experimentally for the range of proportional and nonproportional loading regimes and normalized stress states shown

\subsection{Fatigue life predictions based on critical stored energy density}

Note the number of cycles to crack nucleation is assumed to be approximately equal to the number of cycles to fatigue failure; that is, the number of cycles required for crack propagation through to final fracture is small. The critical stored energy density is determined from the experimentally measured fatigue lives, together with Eqns. (8) and (10), to be $404 \mathrm{~J} / \mathrm{m}^{2}$ and the computed fatigue life predictions using the stored energy criterion are shown in Fig. 13(b). It can be seen that the longest predicted lives occur for pure torsion, consistent with the experiment observations. The predicted fatigue life for in-phase tension-torsion (IP) is shorter 
than that for pure torsion at the given stress level, but higher than that for pure tension loading. The non-proportional square loading path (SQ) leads to the shortest lifetime when the normalized stress level is below 0.85. As in the experiments, the differences in fatigue lives for the various loading types increase with decreasing applied stress. It is apparent that at higher applied Mises stress, the predicted stress-cycles to failure lines would converge as in the experimental observations. These results confirm that the inclusion of the GND evolution in the stored energy calculation leads to the correct ranking of fatigue life, differentiating appropriately for fatigue performance under non-proportional and proportional loading, and also within the loading regimes (ie T, IP, and A in proportional and AT and SQ in nonproportional loading).

\section{Discussion}

In this paper, CPFE modelling with a stored energy criterion is utilized to provide new insights in to the physical and mechanical basis of fatigue lifetime variations under differing loading paths, including axial, torsional, in-phase proportional, out-of-phase non-proportional loading in both 316L stainless steel and nickel RR1000. Increasingly, both experimental and micromechanical studies have indicated that fatigue crack nucleation relies upon the establishment of slip localization for its onset and that it is a necessary condition, but that it is not in its own right sufficient. The present study, which draws together a range of proportional and non-proportional fatigue studies in 316L steel and RR1000, with representative CP modelling, reinforces this. The local stored (elastic) energy density, which can only be approximately determined with modelling at the crystal level, provides a better predictor of the fatigue responses observed and has thrown up some interesting observations.

Considering first simply the proportional axial and torsional loading for a given applied Mises stress, it is apparent from the experimental data in this study (and in the literature) that these loadings give rise to differing fatigue lives, even for the same Mises stress. The stored energies in the two cases are shown to be different, resulting from the slip system groups activated, resulting in differing fatigue lives. For instance, in nickel-based superalloy RR1000, the torsional fatigue gave rise in experiments to longer fatigue lives, for the applied stresses considered, than the axial fatigue tests. This is nicely captured and explained by the cyclic stored energy density.

Many independent studies show that experimental non-proportional axial and torsional loading gives rise to shorter fatigue lives for given Mises stress than for either of the respective proportional axial or torsional loading. The stored energy captures this experimental observation, and it is noted that the cyclic plastic strain also does so. This is argued to result from the higher number of slip systems activated by this loading, leading to the development of additional cyclic hardening, in turn leading to increasing stored energy, earlier fatigue crack nucleation, and shorter fatigue lives under non-proportional loading, which has been reported in many studies. The slip distributions shown in Fig. 8 reinforce this conclusion. There is evidence in the literature [33] that multiple, differing slip system activation, as is argued to occur in non-proportional loading, leads to increases in local GND density. Figure 14 shows multi-slip activation captured using high-resolution digital image correlation in an austenitic steel and the corresponding elevated GND densities at the locations of slip system interactions. This data therefore indicates that high GND density resulting from multi-slip system interaction is a mechanistic driver in non-proportional fatigue by pushing up local stored energy. 
(a)

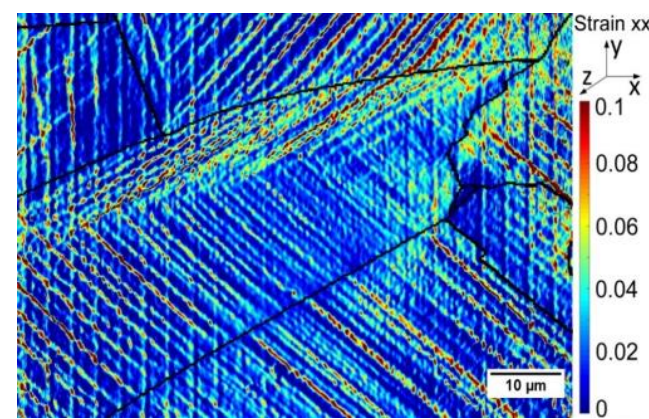

(b)

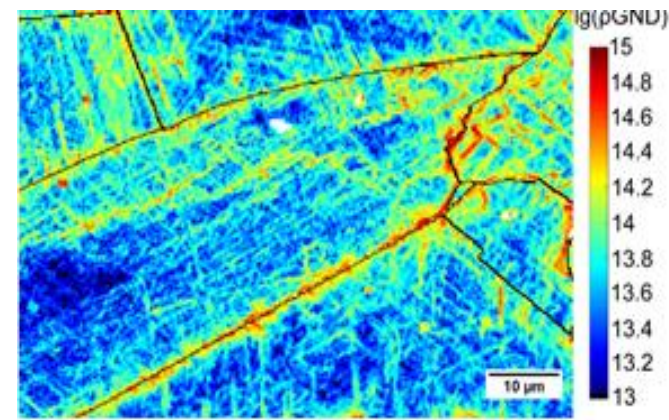

Figure 14. Austenitic steel showing intragranular (a) multi-slip activation from high-resolution digital image correlation and (b) elevated GND density $\left(\mathrm{m}^{-2}\right)$ where the slip systems interact (and at grain boundaries) [33].

The 316L fatigue tests which included small amplitude higher frequency axial or torsional oscillations superposed on base level axial fatigue were found to be mildly detrimental to fatigue life, particularly for relatively lower applied stress levels. The stored energy density captured these observations, also showing that as the applied base axial stress level increased, then so the detriment to fatigue life introduced by the fatigue perturbation decreased. Also, as the magnitude of the fatigue perturbation increased, so the fatigue life was anticipated to drop. Interestingly, for the lower applied stresses for which the effect is most significant, the superposed torsional perturbation fatigue was predicted by stored energy density to be more damaging, and hence detrimental to life, than the axial fatigue perturbation. This transpired to be demonstrated also in the experiments. The superposed perturbed fatigue had two effects in the loading which were to increase moderately the effective amplitude and to introduce a small degree of non-proportionality, both of which are detrimental to fatigue life.

\section{Conclusions}

a) The differing loading states considered (e.g. proportional and non-proportional axial and torsional fatigue) give rise to substantially larger differences in peak stored energies than result from microstructural (morphological) differences in randomly textured polycrystals. Hence stored energy captures both fatigue scatter from microstructural variation and the very different fatigue lives resulting from loading regime stress state.

b) Non-proportional loading is detrimental to fatigue life in 316L steel and RR1000 nickel and results from broader slip system activation, driving elevated stored energy levels. Where GND density evolution is significant (e.g. fine-grained nickel RR1000), fatigue life in non-proportional loading is differentiated from proportional by higher GND densities, which can be generated by slip system interactions, driving up stored energy. The stored energy criterion captures the experimental observations.

c) The effect of non-proportional axial and torsional perturbations on fatigue lifetime is well captured by stored energy density, showing good agreement with the corresponding experimental observations in 316L.

d) A critical stored energy of $592 \mathrm{~J} / \mathrm{m}^{2}$ was extracted for the $316 \mathrm{~L}$ steel considered from axial fatigue data alone enabling good qualitative and reasonable quantitative prediction of fatigue life across the loading regimes considered. The critical energy for nickel alloy RR1000 was found to be $404 \mathrm{~J} / \mathrm{m}^{2}$. 


\section{Acknowledgements}

Bo Chen wishes to acknowledge the financial support provided by Imperial College-China Scholarship Council (IC-CSC), and Fionn Dunne acknowledges the provision of funding for his Royal Academy of Engineering Research Chair. The authors gratefully acknowledge helpful discussions with Dr Duncan Maclachlan, and kind provision of the RR1000 data by Rolls-Royce plc.

\section{References}

1. C. Lu, J. Melendez, and J. Martínez-Esnaola, A universally applicable multiaxial fatigue criterion in 2D cyclic loading. International Journal of Fatigue, 2018. 110: p. 95-104.

2. G. Facheris and K. Janssens, Cyclic mechanical behavior of 316L: uniaxial LCF and strain-controlled ratcheting tests. Nuclear Engineering and Design, 2013. 257: p. 100108.

3. B.-R. You and S.-B. Lee, A critical review on multiaxial fatigue assessments of metals. International Journal of Fatigue, 1996. 18(4): p. 235-244.

4. S. Chattopadhyay, Investigation of proportional and non-proportional loadings using Mohr's circle. 2012.

5. K. Janssens, Proportionally and non-proportionally perturbed fatigue of stainless steel. International Journal of Fatigue, 110: p. 42-48. 2017.

6. Z.-R. Wu, X.-T. Hu, and Y.-D. Song, Multiaxial fatigue life prediction for titanium alloy TC4 under proportional and nonproportional loading. International Journal of Fatigue, 2014. 59: p. 170-175.

7. K. Kanazawa, K. Miller, and M. Brown, Low-cycle fatigue under out-of-phase loading conditions. Journal of Engineering Materials and Technology, 1977. 99(3): p. 222-228.

8. Y. Xiong, Q. Yu, and Y. Jiang, Multiaxial fatigue of extruded AZ31B magnesium alloy. Materials Science and Engineering: A, 2012. 546: p. 119-128.

9. A. Carpinteri, E. Macha, R. Brighenti, et al., Critical fracture plane under multiaxial random loading by means of Euler angles averaging, in European Structural Integrity Society. 1999, Elsevier. p. 166-178.

10. C. Lu, J. Melendez, and J. Martínez-Esnaola, Prediction of crack initiation plane direction in high-cycle multiaxial fatigue with in-phase and out-of-phase loading. Fatigue \& Fracture of Engineering Materials \& Structures, 2017. 40(12): p. 1994-2007.

11. D.-G. Shang, G.-Q. Sun, J. Deng, et al., Multiaxial fatigue damage parameter and life prediction for medium-carbon steel based on the critical plane approach. International Journal of Fatigue, 2007. 29(12): p. 2200-2207.

12. D. Collins, T. Erinosho, F. Dunne, et al., A synchrotron X-ray diffraction study of nonproportional strain-path effects. Acta Materialia, 2017. 124: p. 290-304.

13. F. Rotvel, Biaxial fatigue tests with zero mean stresses using tubular specimens. INTERNAT. J. MECH. SCI., 1970. 12(7): p. 597-613.

14. L. Reis, B. Li, and M. De Freitas, A multiaxial fatigue approach to Rolling Contact Fatigue in railways. International Journal of Fatigue, 2014. 67: p. 191-202.

15. C. Hua and D. Socie, Fatigue damage in 1045 steel under variable amplitude biaxial loading. Fatigue \& Fracture of Engineering Materials \& Structures, 1985. 8(2): p. 101114.

16. S. Kalluri and P. J. Bonacuse, In-phase and out-of-phase axial-torsional fatigue behavior of haynes 188 superalloy at 760 C, in Advances in multiaxial fatigue. 1993, ASTM International.

17. D. F. Socie and G. B. Marquis, Multiaxial fatigue. 2000: Society of Automotive Engineers Warrendale, PA. 
18. D. Shi, J. Huang, X. Yang, et al., Effects of crystallographic orientations and dwell types on low cycle fatigue and life modeling of a SC superalloy. International Journal of Fatigue, 2013. 49: p. 31-39.

19. M. W. Brown and K. Miller, A theory for fatigue failure under multiaxial stress-strain conditions. Proceedings of the Institution of Mechanical Engineers, 1973. 187(1): p. 745-755.

20. A. Fatemi and D. F. Socie, A Critical Plane Approach to Multiaxial Fatigue Damage Including out-of-Phase Loading. Fatigue \& Fracture of Engineering Materials \& Structures, 1988. 11(3): p. 149-165.

21. C. Wang and M. Brown, A path-independent parameter for fatigue under proportional and non-proportional loading. Fatigue $\&$ fracture of engineering materials $\&$ structures, 1993. 16(12): p. 1285-1297.

22. K. Kim and J. Park, Shear strain based multiaxial fatigue parameters applied to variable amplitude loading. International Journal of Fatigue, 1999. 21(5): p. 475-483.

23. M. W. Brown and K. J. Miller, Mode I fatigue crack growth under biaxial stress at room and elevated temperature, in Multiaxial fatigue. 1985, ASTM International.

24. B. Lee, K. Kim, and K. Nam, Fatigue analysis under variable amplitude loading using an energy parameter. International Journal of Fatigue, 2003. 25(7): p. 621-631.

25. M. Noban, H. Jahed, S. Winkler, et al., Fatigue characterization and modeling of 30CrNiMo8HH under multiaxial loading. Materials Science and Engineering: A, 2011. 528(6): p. 2484-2494.

26. R. Branco, P. Prates, J. Costa, F. Berto, A Kovousov, New methodology of fatigue life evaluation for multiaxially loaded notched components based on two uniaxial straincontrolled tests. International Journal of Fatigue, 2018. 111: p. 308-320.

27. F. Berto, P. Gallo, and P. Lazzarin, High temperature fatigue tests of un-notched and notched specimens made of 40CrMoV13. 9 steel. Materials \& Design, 2014. 63: p. 609619.

28. A. Carpinteri, C. Ronchei, D. Scorza, S. Vantadori, Fatigue life estimation for multiaxial low-cycle fatigue regime: the influence of the effective Poisson ratio value. Theoretical and Applied Fracture Mechanics, 2015. 79: p. 77-83.

29. B. Chen, J. Jiang, and F. P. Dunne, Microstructurally-sensitive fatigue crack nucleation in Ni-based single and oligo crystals. Journal of the Mechanics and Physics of Solids, 2017. 106: p. 15-33.

30. B. Chen, J. Jiang, and F. P. Dunne, Is stored energy density the primary meso-scale mechanistic driver for fatigue crack nucleation? International Journal of Plasticity, 2018. 101: p. 213-229.

31. V. Wan, D. MacLachlan, and F.P.E. Dunne, A stored energy criterion for fatigue crack nucleation in polycrystals. International Journal of Fatigue, 2014. 68: p. 90-102.

32. M. A. Groeber and M. A. Jackson, DREAM. 3D: a digital representation environment for the analysis of microstructure in 3D. Integrating Materials and Manufacturing Innovation, 2014. 3(1): p. 5.

33. C. Zhao, D. Stewart, J. Jiang, F.P.E. Dunne, A Comparative Assessment of Iron and Cobalt-based Hard-facing Alloy Deformation using HR-EBSD and HR-DIC. Acta Materialia, 2018. 159: p. 173-186. 